\title{
Optimal power generation for wind-hydro-thermal system using meta-heuristic algorithms
}

\author{
Thuan Thanh Nguyen ${ }^{1}$, Van-Duc Phan ${ }^{2}$, Bach Hoang Dinh ${ }^{3}$, Tan Minh Phan ${ }^{4}$, Thang Trung Nguyen \\ ${ }^{1}$ Faculty of Electrical Engineering Technology, Industrial University of Ho Chi Minh City, Vietnam \\ ${ }^{2}$ Faculty of Automobile Technology, Van Lang University, Vietnam \\ ${ }^{3,5}$ Power System Optimization Research Group, Faculty of Electrical and Electronics Engineering, \\ Ton Duc Thang University, Vietnam \\ ${ }^{4}$ Faculty of Electrical and Electronics Engineering, Ton Duc Thang University, Vietnam
}

\section{Article Info}

Article history:

Received Mar 25, 2019

Revised Apr 27, 2020

Accepted May 8, 2020

\section{Keywords:}

Cuckoo search algorithm

Fitness function

Hydrothermal system

Total fuel cost

Wind turbine

\begin{abstract}
In this paper, cuckoo search algorithm (CSA) is suggested for determining optimal operation parameters of the combined wind turbine and hydrothermal system (CWHTS) in order to minimize total fuel cost of all operating thermal power plants while all constraints of plants and system are exactly satisfied. In addition to CSA, Particle swarm optimization (PSO), PSO with constriction factor and inertia weight factor (FCIW-PSO) and social ski-driver (SSD) are also implemented for comparisons. The CWHTS is optimally scheduled over twenty-four one-hour interval and total cost of producing power energy is employed for comparison. Via numerical results and graphical results, it indicates CSA can reach much better results than other ones in terms of lower total cost, higher success rate and faster search process. Consequently, the conclusion is confirmed that CSA is a very efficient method for the problem of determining optimal operation parameters of CWHTS.
\end{abstract}

Copyright (c) 2020 Institute of Advanced Engineering and Science. All rights reserved.

\section{Corresponding Author:}

Thang Trung Nguyen,

Power System Optimization Research Group,

Faculty of Electrical and Electronics Engineering,

Ton Duc Thang University,

19 Nguyen Huu Tho street, Tan Phong ward, District 7, Ho Chi Minh City, Viet Nam.

Email: nguyentrungthang@tdtu.edu.vn

NOMENCLATURE
$N_{t p}$
$N_{\text {in }}$
$k_{i}, m_{i}, n_{i}$
$P T_{i, j}, P H_{k, j}, P W_{w, j}$
$N_{t p}, N_{h p}, N_{w t}, N_{i n}$
$P_{\text {load }, j}, P_{\text {loss } j}$
$P W_{w}, P W_{w, \text { rate }}$
$W V_{,}, W V_{\text {rate }}, W V_{\text {cut-in }}$,
$W V_{\text {cut-out }}$
$P W_{w, \min }, P W_{w, \text { max }}$
$X_{k}, Y_{k}, Z_{k}$
$Q_{k, \text { min }}, Q_{k, \text { max }}$
$Q_{k, j}$
$W_{\text {avai }, k}$

Number of thermal units

Number of scheduled intervals

Coefficient of fuel cost function

Generation of the ith thermal unit, the kth hydro unit and the wth wind turbine at the jth interval

Number of thermal units, hydro units, wind turbines and intervals.

Power of load and loss at the $j t h$ interval

Generation and rated generation of the wth wind turbine

Wind speed, rated wind speed, cut-in speed and cut-out speed

Minimum and maximum generation of the wth wind turbine coefficients of the kth hydro unit's generation Minimum and maximum discharge of the kth hydro unit Discharge of the kth hydro unit at the jth interval available water for power generation over the scheduled intervals 


\section{INTRODUCTION}

Hydrothermal system scheduling (HTSS) problem is a very important problem in optimization operation of power systems where hydropower plants and thermal plants are accounting for a high rate of all power sources in exiting power systems [1]. In general, hydropower plants use water in river to drive turbines and run generators for producing electricity to loads while thermal power plants must employ fossil fuel such as gas, oil and coal to drive gas turbines or steam turbines for generating electricity. Water can be exhausted and full in rivers dependent on weather, namely rain and sun in seasons [2]. On the contrary, fossil fuels cannot be recovered after using. As a result, cost of generating electricity or price of fossil fuels in thermal power plants is a significant issue but cost of water in hydropower plants is normally ignored. Main issues regarding hydropower plants are hydraulic constraints such as discharge limit, spillage, flood, and reservoir limits. So, in hydropower system scheduling problem, the most difficulty issue is to solve the hydraulic constraints successfully while the main target is to reduce cost of generating electricity in thermal power plants [3].

Basically, hydrothermal system scheduling problem can be divided into short-term [1-10], medium-term [11-15] and long-term models [16-20] based on the time period of scheduled optimization. Short-term HTSS problem is classified into fixed-head model [1-7] and variable head model [8-10], and this problem was also the most attracted problem among three different time period types. The main difference of the problems is scheduled time period. Short-term HTSS problem considers one day to one week while long-term HTSS considers over one year with twelve months or four seasons. The time from one week to one month or from one month to one season is taken into account in medium-term HTSS problem. The three problems have the same characteristic that is to consider cost of producing electricity in thermal power plants as an objective and neglect cost in hydropower plants. In addition, renewable energies like solar energy and wind energy are not considered in the problem.

In recent years, wind turbines have been considered in conventional power systems with hydrothermal plants. The optimal generation between these thermal plants and these wind turbines was successfully solved by using metaheuristic algorithms like bee colony algorithm (BCA) [21] and Wait-See algorithm (WSA) [22]. Then, the integrated system was expanded by adding hydropower plants and the optimal generation of the wind-hydro-thermal system were solved by using nondominated sorting genetic algorithm-III (NSGA-III) [23], multi-objective bee colony optimization algorithm (MOBCOA) [24], two-stage stochastic method (TSSM) [25] and sine-cosine algorithm (SCA) [26]. In [23], multi objective functions including fuel cost and power loss are considered in which power generation of wind farms is considered as a control variable of the combined system. In [24], uncertainty of wind speed was considered by considering Weibull distribution function. In the study, wind turbines are calculated three cost, direction cost, reserve cost and penalty cost. In [25-26], cascaded hydropower plants are considered together with the power generation of thermal power plants and wind turbines. Similar to [24], the two studies also considered the Weibull function and three costs of wind turbines. In general, almost all studies applied metaheuristics and mainly focused on the highly successful constraint handling ability of rather than reaching the best solutions for the problem. In addition, power loss of the system due to the impact of resistance and reactance of conductors was not considered in these studies. This is also understood because these studies were first application of methods for solving the new problem.

In this paper, short-term HTSS problem with fixed-head model is expanded by adding wind turbines and considering operation range of them. On the contrary to other studies, all constraints of hydropower plants are taken into account including discharge limit, available water and generator limits. Thermal power plants are not constrained by available fossil fuel quantity but generators. Wind turbines are constrained by capacity and operation wind speeds. The main purpose is to calculate cost of thermal power plants and determine the most optimal generation for reducing this cost. For reaching the optimal solutions of the problem, we apply PSO (PSO) [27], CFIWPSO [28], SSD [29] and CSA [30].

In summary, the contributions of the paper are follows:

- Develop wind-hydrothermal system scheduling problem with short-term model

- Propose the best decision variable selection method

- Investigate performance of PSO, FCIW-PSO, SSD and CSA 


\section{FORMULATION OF OPTIMAL SCHEDULING OF WIND-HYDRO-THERMAL SYSTEM}

In the section, a wind-hydrothermal system with fixed head model is in detail described by using figure and formulas. Figure 1 shows a system with one thermal power plant, one hydropower plant and one wind farm located at load. The objective and constraints as well as assumption of the problem are as follows:

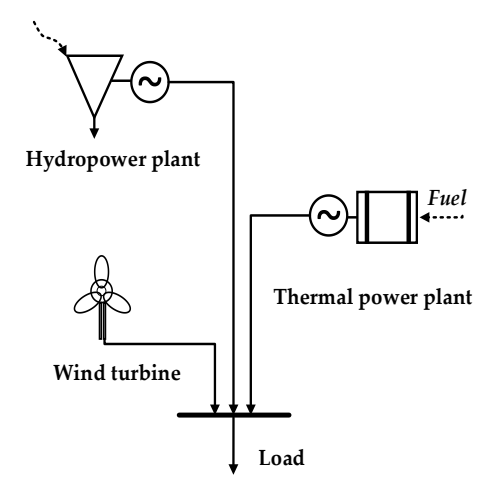

Figure 1. A typical wind-hydro-thermal system

\subsection{Objective function}

Total fuel cost (TFC) for generating electricity from all thermal power plants is considered as a major part that needs to be minimized as much as possible. The objective is shown as follows:

$$
\text { Minimize TFC }=\sum_{i=1}^{N_{t p}} \sum_{j=1}^{N_{\text {in }}}\left(k_{i}+m_{i} P T_{i, j}+n_{i}\left(P T_{i, j}\right)^{2}\right)
$$

In (1), we only focus on the reduction of fuel cost from thermal power plants meanwhile the electric generation cost from hydropower plants and wind power plants is neglected. The assumption of neglecting the electric cost from hydroelectric plant is taken from the idea that water is a nature source with very low price whereas all power energy from wind power plants is absolutely used with the same price and the same cost over one scheduled day.

\subsection{The set of constraints}

a. Constraints from power system

In power systems, the balance between the generated and consumed power must be guaranteed as the following model:

$$
\sum_{i=1}^{N_{t p}} P T_{i, j} \sum_{k=1}^{N_{h p}} P H_{k, j}+\sum_{w=1}^{N_{w t}} P W_{w, j}-P_{L o a d, j}-P_{\text {Loss }, j}=0
$$

b. Constraint from thermal plants

Power generation of thermal power plants is limited as follows:

$$
P T_{i, \min } \leq P T_{i, j} \leq P T_{i, \max }
$$

c. Constraint from wind turbines

Basically, power generation of a wind turbine is much dependent on wind speed. The range of generation can be seen by the following equation [25]:

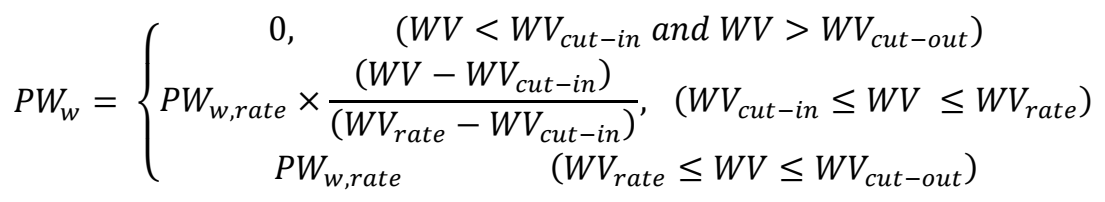


So, wind turbines are also constrained by power generation as follows:

$$
P W_{w, \min } \leq P W_{w, j} \leq P W_{w, \max }
$$

d. Constraints from hydropower plants:

Limits of water Discharge: Water that is discharged through a turbine must be in a predetermined range as follows:

$$
Q_{k, \min } \leq Q_{k, j} \leq Q_{k, \max }
$$

where $Q_{k, j}$ is determined as follows:

$$
Q_{k, j}=X_{k}+Y_{k} P H_{k, j}+Z_{k}\left(P H_{k, j}\right)^{2}
$$

In addition, the total water discharge over $N_{\text {in }}$ intervals must be equal to available as the constraint below:

$$
\sum_{j=1}^{N_{\text {in }}} Q_{k, j}=W_{\text {avai }, k}
$$

e. Constraint of generators: Hydro generation is constrained by.

$$
P H_{k, \min } \leq P H_{k, j} \leq P H_{k, \max }
$$

\section{CUCKOO SEARCH ALGORITHM}

\subsection{New solution generation mechanism}

On the contrary to PSO and SSD, CSA performs two mechanisms to produce new solutions. For each mechanism, the whole population is newly updated. So, total new solutions generated by CSA is two times that of PSO and SSD. Lévy flights is applied in the first mechanism while mutation operation is employed in the second one. The two mechanisms are mathematically formulated as follows:

$$
\begin{aligned}
& \operatorname{Sol}_{x}^{\text {new }}=\operatorname{Sol}_{x}+\alpha_{0}\left(\operatorname{Sol}_{x}-\operatorname{Sol}_{B e s t}\right) \otimes L(\beta) \\
& \text { Sol }_{x}^{\text {new }}= \begin{cases}\operatorname{Sol}_{x}+r d_{1}\left(\operatorname{Sol}_{1}-\operatorname{Sol}_{2}\right) & \text { if } r d_{2}<\text { Pro } \\
\operatorname{Sol}_{x} & \text { otherwise }\end{cases}
\end{aligned}
$$

where $\alpha_{0}$ is a positive scaling factor; $\mathrm{L}(\beta)$ is Lévy distribution function [10]; and Sol $_{\text {Best }}$ is the so-far best solution among the current population; $r d_{1}$ and $r d_{2}$ are random numbers in the range between 0 and 1; Pro is old solution replacement probability, which is selected within 0 and 1 . $\mathrm{Sol}_{1}$ and $\mathrm{Sol}_{2}$ are two randomly selected solutions.

\subsection{Promising solution selection mechanism}

This mechanism is applied to performance comparison of quality between the new xth solution and the old xth solution to retain a better solution and abandon a worse one. So, fitness function must be calculated for each old and new solution. Then, the following model is applied.

$$
\text { Sol }_{x}= \begin{cases}\text { Sol }_{x} & \text { if Fit } \\ \text { Sol }_{x}^{\text {new }} & \text { Otherwise }\end{cases}
$$

\section{RESULTS NUMERICAL RESULTS}

In this section, the effectiveness of CSA is compared to that of PSO, CFIW-PSO and SSD on the system with one thermal power plant, one hydropower plant and one wind power plant. The system is scheduled over twenty-four one-hour intervals. The hydrothermal systems and loss coefficients are taken 
from Table A1 in page 284 [10] while the wind farm data is taken from wind farm 1 in Table 6 in page 760 [31]. The whole data and loss coefficients are shown in Table A1, Table A2 and Table A3 in Appendix. The four methods are coded on Matlab program language and a computer with CPU of Intel Core i7-2.4GHz-RAM 4GB for obtaining 50 successful runs.

In order to run these methods, population size $(P S)$ and the maximum iteration $(M I)$ are set to 20 and 2000 for CSA, 40 and 2000 for PSO, CFIW-PSO and SSD. The results from 50 successful runs are summarized in Table 1 in addition to saving cost and improvement shown in Figures 2 and 3. In the two figures, saving cost and the corresponding improvement level of CSA as compared to PSO, CFIW-PSO and SSD are shown. So, there is no bar to show the result of CSA in the two figures. From the figures, it can indicate that as compared to other methods CSA can reach very high reduction of minimum cost with $\$ 6029.58$, mean cost with $\$ 7576.37$ and maximum cost with $\$ 9305.77$ the reduction cost of CSA is corresponding to the improvement level of $8 \%, 0.94 \%$ and $2.1 \%$ over PSO, CFIW-PSO and SSD. Similarly, the mean cost and the highest cost of CSA are also much less than other methods. The improvement level of mean cost and the highest cost can be up to $4 \%$ and $9.8 \%$.

Table 1. Summary of results

\begin{tabular}{lllll}
\hline & PSO & CFIW-PSO & SSD & CSA \\
\hline Minimum cost (\$) & 75789.64 & 70420.13 & 71236.93 & 69760.06 \\
Average cost (\$) & 77362.83 & 72718.63 & 73327.8 & 69786.46 \\
Maximum cost (\$) & 79306.06 & 75847.33 & 77212.04 & 70000.29 \\
Standard deviation $(\$)$ & 729.5481 & 1438.077 & 1366.289 & 41.4461 \\
Success rate $(\%)$ & $848 / 50$ & $86 / 50$ & $107 / 50$ & $50 / 50$ \\
\hline
\end{tabular}

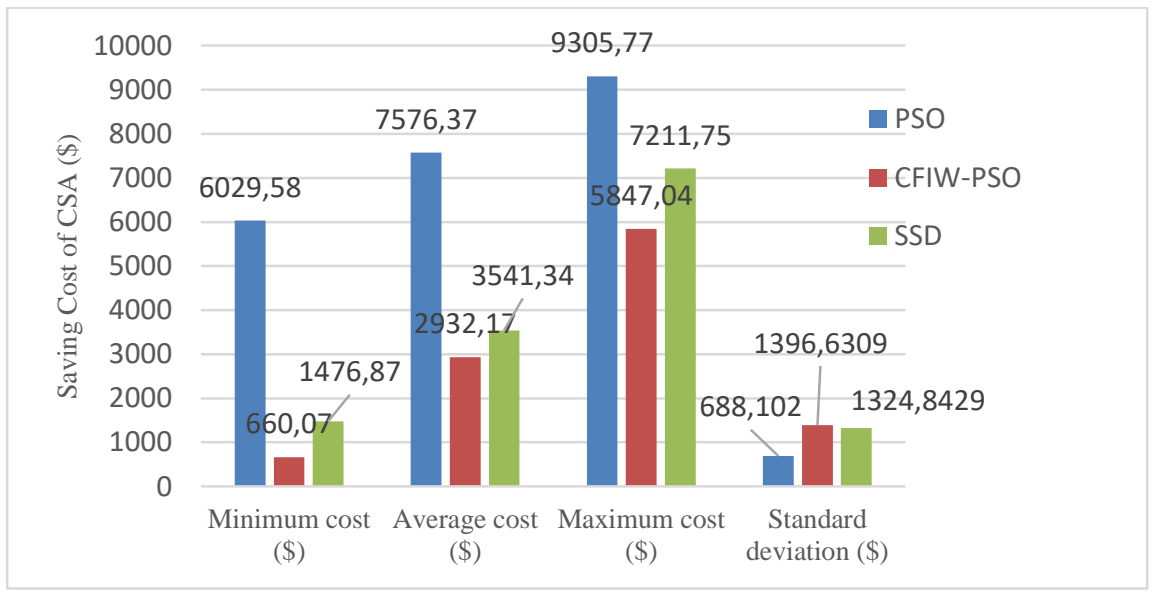

Figure 2. Saving cost of CSA as compared to PSO, CFIW-PSO and SSD

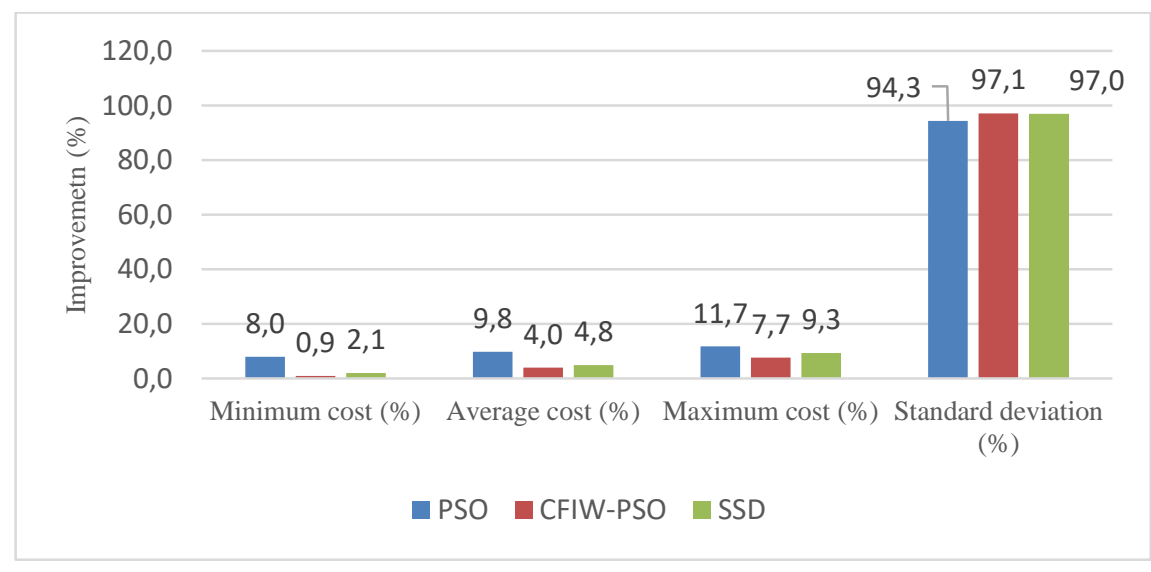

Figure 3. Improvement of CSA over PSO, CFIW-PSO and SSD 
In addition, the best run, the mean run, the worst run and the cost of 50 runs can be observed from Figures 4-7. The figures indicate that CSA is always the best method with the fastest speed and all better runs. Consequently, it leads to a conclusion that CSA is the best method for the first system. Optimal power generation obtained by CSA is shown in Figure 8.

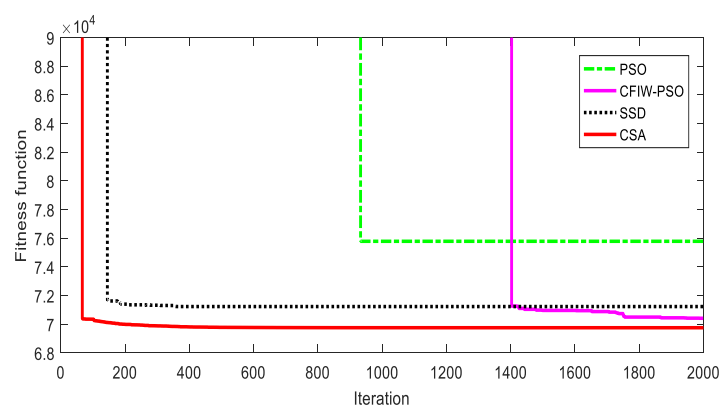

Figure 4. The best convergence characteristic of four applied methods

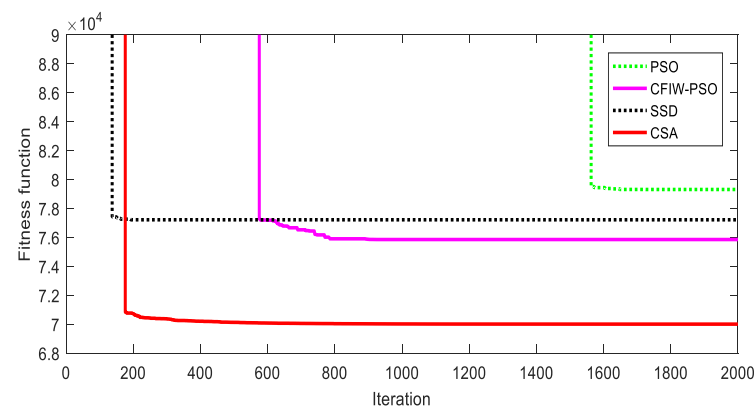

Figure 6. The worst convergence characteristic of four applied methods

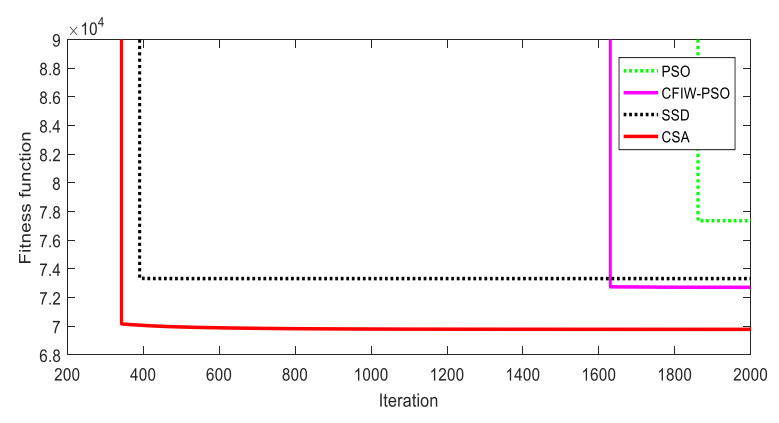

Figure 5. The mean convergence characteristic over 50 successful runs of four applied methods

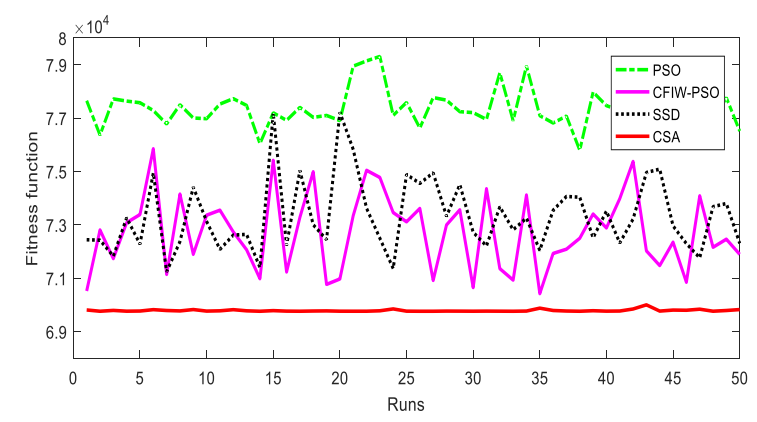

Figure 7. Fuel cost of 50 successful runs obtained by four applied methods

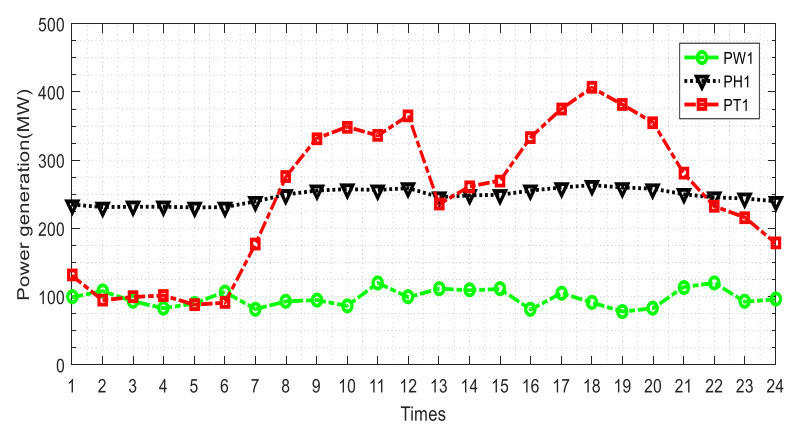

Figure 8. Optimal power generation obtained by CSA

\section{CONCLUSION}

In this paper, four applied methods including CSA, PSO, FCIW-PSO and SSD have been applied for solving combined wind turbine and hydrothermal systems. The four method have been implemented for reaching 50 successful runs for comparisons. Numerical results including the best cost, mean cost and maximum cost in addition to graphical results including convergence characteristics have been analyzed for evaluating performance of these methods. CSA was superior to three other ones in finding the best solution, reach very high success rate and faster speed. So, it can be concluded that CSA is a very efficient method for determining optimal parameters of combined wind turbines and hydrothermal systems. 


\section{APPENDIX}

Table A1. Data of thermal power plant

\begin{tabular}{ccccc}
\hline$k_{l}$ & $m_{l}$ & $n_{l}$ & $P T_{l, \min }(\mathrm{MW})$ & $P T_{1, \max }(\mathrm{MW})$ \\
\hline 373.7 & 9.606 & 0.001991 & 0 & 505 \\
\hline
\end{tabular}

Table A2. Data of hydroelectric plant

\begin{tabular}{cccccc}
\hline$X_{I}$ & $Y_{1}$ & $Z_{l}$ & $W_{\text {avai, }}$ & $P H_{1, \min }(\mathrm{MW})$ & $P H_{1, \max }(\mathrm{MW})$ \\
\hline 61.53 & -0.009079 & 0.0007749 & 2559.6 & 0 & 300 \\
\hline
\end{tabular}

The loss coefficient matrix of the system

$$
B=\left[\begin{array}{ll}
0.00005 & 0.00001 \\
0.00001 & 0.00015
\end{array}\right]
$$

Table A3. Load and wind power over 24 one-hour intervals

\begin{tabular}{ccccccccc}
\hline$j$ & $P_{\text {Load }, j}$ & $P W_{1, j}$ & $j$ & $P_{\text {Load }, j}$ & $P W_{1, j}$ & $j$ & $P_{\text {Load }, j}$ & $P W_{1, j}$ \\
\hline 1 & 455 & 99 & 9 & 665 & 94.8 & 17 & 721 & 705 \\
2 & 425 & 108 & 10 & 675 & 86.4 & 18 & 740 & 91.2 \\
3 & 415 & 93 & 11 & 695 & 120 & 19 & 700 & 78 \\
4 & 407 & 82.8 & 12 & 705 & 99 & 20 & 678 & 82.8 \\
5 & 400 & 90 & 13 & 580 & 111.6 & 21 & 630 & 114 \\
6 & 420 & 106.8 & 14 & 605 & 109.2 & 22 & 585 & 120 \\
7 & 487 & 81.6 & 15 & 616 & 111 & 23 & 540 & 92.4 \\
8 & 604 & 93 & 16 & 653 & 81 & 24 & 503 & 96 \\
\hline
\end{tabular}

\section{REFERENCES}

[1] A. J. Wood, B. F. Wollenberg, G. B. Sheblé, "Power generation, operation, and control," John Wiley \& Sons, 2013.

[2] I. A. Farhat, M. E. El-Hawary, "Fixed-head hydro-thermal scheduling using a modified bacterial foraging algorithm," In 2010 IEEE Electrical Power \& Energy Conference IEEE, pp. 1-6, Aug. 2010.

[3] J. Sasikala, and M. Ramaswamy, "Optimal gamma based fixed head hydrothermal scheduling using genetic algorithm," Expert Systems with Applications, vol. 37, no. 4, pp. 3352-3357, 2010.

[4] M. Y. Chang and W. J. A. Huang, "A Practical Case Report on the Node Point of a Butterfly Model Circular Economy: Synthesis of a New Hybrid Mineral-Hydrothermal Fertilizer for Rice Cropping," Sustainability, vol. 12, no. 3, pp. 1-8, 2020.

[5] N. Hamzah, K. Tokimatsu, and K. Yoshikawa, "Solid fuel from oil palm biomass residues and municipal solid waste by hydrothermal treatment for electrical power generation in Malaysia: a review," Sustainability, vol. 11, no. 4, pp. 1-23, 2019.

[6] S. Ghosh, M. Kaur, S. Bhullar, and V. Karar, "Hybrid abc-bat for solving short-term hydrothermal scheduling problems," Energies, vol. 12, no. 3, pp. 1-15, 2019.

[7] T. T. Nguyen, N. Vu Quynh, M. Q. Duong, L. Van Dai, "Modified differential evolution algorithm: A novel approach to optimize the operation of hydrothermal power systems while considering the different constraints and valve point loading effects," Energies, vol. 11, no. 3, pp. 1-30, 2018.

[8] S. K. Damodaran, T. K. Sunil Kumar, "Hydro-thermal-wind generation scheduling considering economic and environmental factors using heuristic algorithms," Energies, vol 11, no. 2, pp. 1-19, 2018.

[9] T. T. Nguyen, B. H. Dinh, N. V. Quynh, M. Q. Duong, M. Q, L. V. Dai, “A novel algorithm for optimal operation of hydrothermal power systems under considering the constraints in transmission networks," Energies, vol. 11, no. 1, pp. 1-21, 2018.

[10] T. T. Nguyen, D. N. Vo, and A. V. Truong, "Cuckoo search algorithm for short-term hydrothermal scheduling," Applied Energy, vol. 132, no. 1, pp. 276-287, 2014.

[11] R. Fuentes-Loyola, V. H. Quintana, "Medium-term hydrothermal coordination by semidefinite programming," IEEE Transactions on Power Systems, vol. 18, no. 4, pp. 1515-1522, 2013.

[12] V. H. Ferreira, and G. H. C. Silva, "Natural optimization applied to medium-term hydrothermal coordination," In 2011 16th International Conference on Intelligent System Applications to Power Systems, pp. 1-6, Sep. 2011.

[13] K. I. Ourani, C. G. Baslis, and A. G. Bakirtzis, "A Stochastic Dual Dynamic Programming model for medium-term hydrothermal scheduling in Greece," In 2012 47th International Universities Power Engineering Conference (UPEC), pp. 1-6, Sep. 2012.

[14] F. Beltrán, W. de Oliveira, E. C. Finardi, "Application of scenario tree reduction via quadratic process to mediumterm hydrothermal scheduling problem," IEEE Transactions on Power Systems, pp. 1-10, 2017. 
[15] M. I. Ennes, A. L. Diniz, "An efficient equivalent thermal cost function model for nonlinear mid-term hydrothermal generation planning," International Journal of Electrical Power \& Energy Systems, vol. 63, pp. 705-712, 2014.

[16] R. W. Ferrero, J. F. Rivera, and S. M. Shahidehpour, "A dynamic programming two-stage algorithm for long-term hydrothermal scheduling of multireservoir systems," IEEE Transactions on Power Systems, vol. 13, no. 4, pp. 1534-1540, 1998.

[17] L. Martinez, S. Soares, "Comparison between closed-loop and partial open-loop feedback control policies in long term hydrothermal scheduling," IEEE Transactions on Power Systems, vol 17, no. 2, pp. 330-336, 2002.

[18] T. Homem-de-Mello, V. L. De Matos, and E. C. Finardi, "Sampling strategies and stopping criteria for stochastic dual dynamic programming: a case study in long-term hydrothermal scheduling," Energy Systems, vol. 2, pp. 1-31, 2011.

[19] G. Da Cruz, and S. Soares, "Non-uniform composite representation hydroelectric systems for long-term hydrothermal scheduling," IEEE Transactions on Power Systems, vol. 11, no. 2, pp. 702-707, 1996.

[20] M. Zambelli, T. G. Siqueira, M. Cicogna, and S. Soares, "Deterministic versus stochastic models for long term hydrothermal scheduling," In 2006 IEEE Power Engineering Society General Meeting, pp. 7, Jun. 2006.

[21] H. T. Jadhav, and R. Roy, "Gbest guided artificial bee colony algorithm for environmental/economic dispatch considering wind power,” Expert Systems with Applications, vol. 40, no. 16, pp. 6385-6399, 2013.

[22] X. Liu, "Economic load dispatch constrained by wind power availability: A wait-and-see approach," IEEE Transactions on Smart Grid, vol. 1, no. 3, pp. 347-355, 2010.

[23] X. Yuan, H. Tian, Y. Yuan, Y. Huang, R. M. Ikram, "An extended NSGA-III for solution multi-objective hydro-thermal-wind scheduling considering wind power cost," Energy Conversion and Management, vol. 96, pp. 568-578, 2015.

[24] J. Zhou, P. Lu, Y. Li, C. Wang, L. Yuan, and L. Mo, "Short-term hydro-thermal-wind complementary scheduling considering uncertainty of wind power using an enhanced multi-objective bee colony optimization algorithm," Energy conversion and management, vol. 123, pp. 116-129, 2016.

[25] M. Daneshvar, B. Mohammadi-Ivatloo, K. Zare, S. Asadi, "Two-stage stochastic programming model for optimal scheduling of the wind-thermal-hydropower-pumped storage system considering the flexibility assessment," Energy, vol. 193, 116657, 2020.

[26] K. Dasgupta, P. K. Roy, and V. Mukherjee, "Power flow based hydro-thermal-wind scheduling of hybrid power system using sine cosine algorithm," Electric Power Systems Research, vol. 178, 106018, 2020.

[27] T. T. Nguyen, and N. D. Vo, "Improved particle swarm optimization for combined heat and power economic dispatch," Scientia Iranica, vol. 23, no. 3, pp. 1318-1334, 2016.

[28] L. C. Kien, T. L. Duong, V. D. Phan, and T. T. Nguyen, "Maximizing Total Profit of Thermal Generation Units in Competitive Electric Market by Using a Proposed Particle Swarm Optimization," Sustainability, vol. 12, no. 3, pp. 1265, 2020.

[29] A. Tharwat, T. Gabel, "Parameters optimization of support vector machines for imbalanced data using social ski driver algorithm," Neural Computing and Applications, pp. 1-14, 2019.

[30] X. Meng, J. Chang, X. Wang, and Y. Wang, "Multi-objective hydropower station operation using an improved cuckoo search algorithm," Energy, vol. 168, pp. 425-439, 2019.

[31] H. Zhang, D. Yue, X. Xie, C. Dou, F Sun, "Gradient decent based multi-objective cultural differential evolution for short-term hydrothermal optimal scheduling of economic emission with integrating wind power and photovoltaic power," Energy, vol. 122, pp. 748-766, 2017. 